\title{
Ultrasonic measurements of the elastic moduli of liquid crystalline polymers
}

\author{
C. L. Choy* and W. P. Leung \\ Department of Physics, The Chinese University of Hong Kong, Hong Kong \\ and A. F. Yee \\ Department of Materials Science and Engineering, University of Michigan, \\ Ann Arbor, MI 48109, USA \\ (Received 24 September 1991; revised 13 December 1991)
}

\begin{abstract}
The five independent elastic moduli of extruded rods of two thermotropic liquid crystalline polymers, Vectra B900 and Vectra A950, and the nine independent moduli of an injection-moulded bar of Vectra A950 have been measured by ultrasonic methods at $10 \mathrm{MHz}$. For Vectra $\mathrm{B} 900$, the sharp rise in the axial extensional modulus $C_{33}$ and the moderate drop in the transverse modulus $C_{11}$ with increasing draw ratio result from the alignment of molecular chains along the extrusion direction. The chain orientation also leads to an anisotropy in the shear modulus, with the axial shear modulus $\left(C_{44}\right)$ exceeding the transverse shear modulus $\left(C_{66}\right)$ by $>50 \%$. The mechanical anisotropy in the surface layer of the injection-moulded bar implies that the chains lie mostly in the plane parallel to the wide surface of the bar, and preferentially along the injection direction. The weak anisotropy in the core indicates almost random molecular orientation. Based on the aggregate model, the upper (Voigt) and lower (Reuss) bounds of the elastic modulus of isotropic Vectra B 900 have been calculated by using the modulus data of the oriented samples. The observed modulus of isotropic Vectra B900 lies within these bounds and is quite close to the lower bound.
\end{abstract}

(Keywords : elastic moduli; thermotropic liquid crystalline polymers)

\section{INTRODUCTION}

There are two routes for the production of ultra high modulus polymers. In the first approach, flexible chain polymers (such as high density polyethylene and polyoxymethylene) are converted into highly oriented, extended chain structures by drawing or extrusion in the solid state. The second approach involves the molecular design of stiff rod-like molecules that show liquid crystalline behaviour in solution (lyotropic) or in the melt (thermotropic) and form oriented extended chain structures in the solid state. Two important examples are the lyotropic aramid, Kevlar, and thermotropic aromatic copolyesters.

Since the first report of the successful preparation of ultra high modulus flexible chain polymers, there has been a large number of studies on the elastic modulus of these materials ${ }^{1-3}$. Since these materials are highly anisotropic, it is important to provide a complete characterization of the elastic anisotropy by measuring all five independent stiffness constants $C_{11}, C_{13}, C_{33}, C_{44}$ and $C_{66}$. For highly drawn samples (normally in the form of long, thin rods or tapes), the ideal method for the determination of $C_{p q}$ is the ultrasonic technique, and we have used it to study the elastic anisotropy of ultra drawn high density polyethylene $e^{4,5}$, polyoxymethylene $e^{6-8}$ and polypropylene ${ }^{6,9}$. In the present work, we apply this method to measure the five independent stiffnesses of extruded rods of two thermotropic liquid crystalline polymers (Vectra A950 and Vectra B900), and the nine

* Now at the Department of Applied Physics, Hong Kong Polytechnic, Hung Hom, Kowloon, Hong Kong independent stiffnesses of an injection-moulded bar of Vectra A950.

\section{EXPERIMENTAL}

\section{Sample preparation}

The starting materials are Vectra A950 and Vectra B900 (Hoechst-Celanese Co.). Vectra A950 is an aromatic copolyester that is believed to consist of $70 \mathrm{~mol} \%$ p-hydroxybenzoic acid (HBA) and $30 \mathrm{~mol} \%$ of $2,6-$ hydroxynaphthoic acid (HNA) and Vectra B900 is an aromatic polyester-amide comprising $58 \mathrm{~mol} \% \mathrm{HNA}$, $21 \mathrm{~mol} \%$ terephthalic acid and $21 \mathrm{~mol} \%$ 4'-hydroxyacetanilide $^{10}$. The processing equipment for extruding Vectra $B 900$ rods has been described in detail previously ${ }^{11}$. Briefly, a Brown 3/4" single screw extruder equipped with a die of length $9.5 \mathrm{~mm}$ and diameter $3.2 \mathrm{~mm}$ was used. The die temperature was kept at $321^{\circ} \mathrm{C}$. Rods of different diameters were prepared by varying the speed of a set of take-up rolls. The draw ratio $\lambda$ was defined as (die diameter/rod diameter) ${ }^{2}$. Extruded rods $(\lambda=3)$ and injection-moulded type 1 tensile bars of Vectra A950 were supplied by Hoechst-Celanese Co. The density of all the samples was measured by the flotation method.

\section{Ultrasonic measurements}

The five independent stiffnesses of the extruded rods were determined by the contact method previously described $^{8}$. A PZT ceramic transducer, bonded to one surface of the sample, generated a beam of pulsed $10 \mathrm{MHz}$ elastic wave that was subsequently received by another transducer bonded to the opposite parallel surface. The 
transit time of the ultrasonic pulse was measured on a gated time interval counter with an accuracy of $1 \mathrm{~ns}$, and the wave velocity was calculated.

If we take the extrusion direction as the $\hat{3}$-axis and denote the velocity by $v_{a b}$, where $a$ and $b$ refer, respectively, to the direction of polarization and propagation of the elastic wave, then the stiffnesses $C_{11}, C_{33}$, $C_{55}$ and $C_{66}$ can be deduced from the following equations :

$$
\begin{aligned}
& C_{11}=\rho v_{11}^{2} \\
& C_{33}=\rho v_{33}^{2} \\
& C_{55}=\rho v_{13}^{2} \\
& C_{66}=\rho v_{12}^{2}
\end{aligned}
$$

where $\rho$ is the density of the sample. The cross-plane stiffness $C_{13}$ can be calculated from $v_{\mathrm{rs}}$, the velocity of the shear wave propagating at $45^{\circ}$ to the extrusion direction:

$$
\begin{aligned}
4 \rho v_{\mathrm{rs}}^{2}= & \left(C_{11}+C_{33}+2 C_{55}\right) \\
& -\left[\left(C_{33}-C_{11}\right)^{2}+4\left(C_{13}+C_{55}\right)^{2}\right]^{1 / 2}(1 \mathrm{e})
\end{aligned}
$$

where $\hat{r}=(1 / \sqrt{2})(1,0,-1)$ and $\hat{s}=(1 / \sqrt{2})(1,0,1)$.

The injection-moulded sample is in the form of a tensile bar $(3.2 \mathrm{~mm}$ thick and $12.3 \mathrm{~mm}$ wide in the central region ). In the central parallel-sided region, the molecular orientation has a simpler pattern and the elastic modulus is independent of the position along the length. Therefore, our measurements were confined to this region. The skin layer is expected to have a higher degree of chain alignment than the core, since the orientation induced by the shear flow is immediately frozen in upon contact with the mould. The skin naturally extended around the whole section, so the width of the bar was trimmed down to $4 \mathrm{~mm}$ ( $\pm 2 \mathrm{~mm}$ from the central line) to leave a section in which the skin regions were located near the top and bottom surfaces. Samples $0.7 \mathrm{~mm}$ thick were then cut from the surface and middle layers for ultrasonic measurements.

The injection-moulded sample has orthorhombic symmetry and requires nine independent stiffnesses for a complete characterization. In addition to the five stiffnesses given in equation (1), two more were obtained from the contact method:

$$
\begin{aligned}
& C_{22}=\rho v_{22}^{2} \\
& C_{44}=\rho v_{32}^{2}
\end{aligned}
$$

Subscripts 1, 2 and 3 refer to the directions along the width, thickness and length of the bar. It was not possible to determine $C_{12}$ and $C_{23}$ by the contact method since the layer was too thin to allow the preparation of samples with sufficiently large dimensions along the wave propagation direction. The immersion method $^{6}$ was used instead. The accuracy of $C_{11}, C_{22}, C_{33}, C_{44}, C_{55}$ and $C_{66}$ is $4 \%$, while that for $C_{12}, C_{13}$ and $C_{23}$ is $8 \%$. Young's moduli calculated from the elastic stiffnesses $\left(C_{\mathrm{pq}}\right)$ have

\begin{tabular}{|c|c|c|c|c|c|c|}
\hline & \multicolumn{4}{|c|}{ Injection-moulded } & & \\
\hline & \multicolumn{2}{|c|}{ Skin } & \multicolumn{2}{|c|}{ Core } & \multicolumn{2}{|c|}{ Extruded $(\lambda=3)$} \\
\hline & $C_{\mathrm{pq}}{ }^{a}$ & $S_{\mathrm{pq}}{ }^{a}$ & $C_{\mathrm{pq}}$ & $S_{\mathrm{pq}}$ & $C_{\mathrm{pq}}$ & $S_{\mathrm{pq}}$ \\
\hline 11 & 9.45 & 0.200 & 11.8 & 0.145 & 8.34 & 0.267 \\
\hline 22 & 7.73 & 0.241 & 10.1 & 0.175 & & \\
\hline 33 & 35.0 & 0.0357 & 12.0 & 0.146 & 52.8 & 0.0219 \\
\hline 44 & 1.69 & 0.592 & 2.01 & 0.498 & 1.80 & 0.555 \\
\hline 55 & 2.74 & 0.365 & 2.28 & 0.439 & & \\
\hline 66 & 1.22 & 0.820 & 1.96 & 0.510 & 1.10 & 0.909 \\
\hline 12 & 5.7 & -0.132 & 6.3 & -0.058 & 6.1 & -0.187 \\
\hline 13 & 7.5 & -0.0179 & 6.8 & -0.051 & 7.2 & -0.010 \\
\hline 23 & 6.6 & -0.0172 & 6.5 & -0.062 & & \\
\hline
\end{tabular}
an error of $6 \%$.

\section{RESULTS AND DISCUSSION}

\section{General features}

The stiffnesses $\left(C_{\mathrm{pq}}\right)$ and compliances $\left(S_{\mathrm{pq}}\right)$ of Vectra A950 are given in Table 1 , and the stiffnesses and Young's
Table 1 Elastic stiffnesses $\left(C_{\mathrm{pq}}\right)$ and compliances $\left(S_{\mathrm{pq}}\right)$ of liquid crystalline polymer Vectra $\mathrm{A} 950$ at $23^{\circ} \mathrm{C}$

${ }^{a}$ The units of $C_{\mathrm{pq}}$ and $S_{\mathrm{pq}}$ are $\mathrm{GPa}$ and $\mathrm{GPa}^{-1}$, respectively

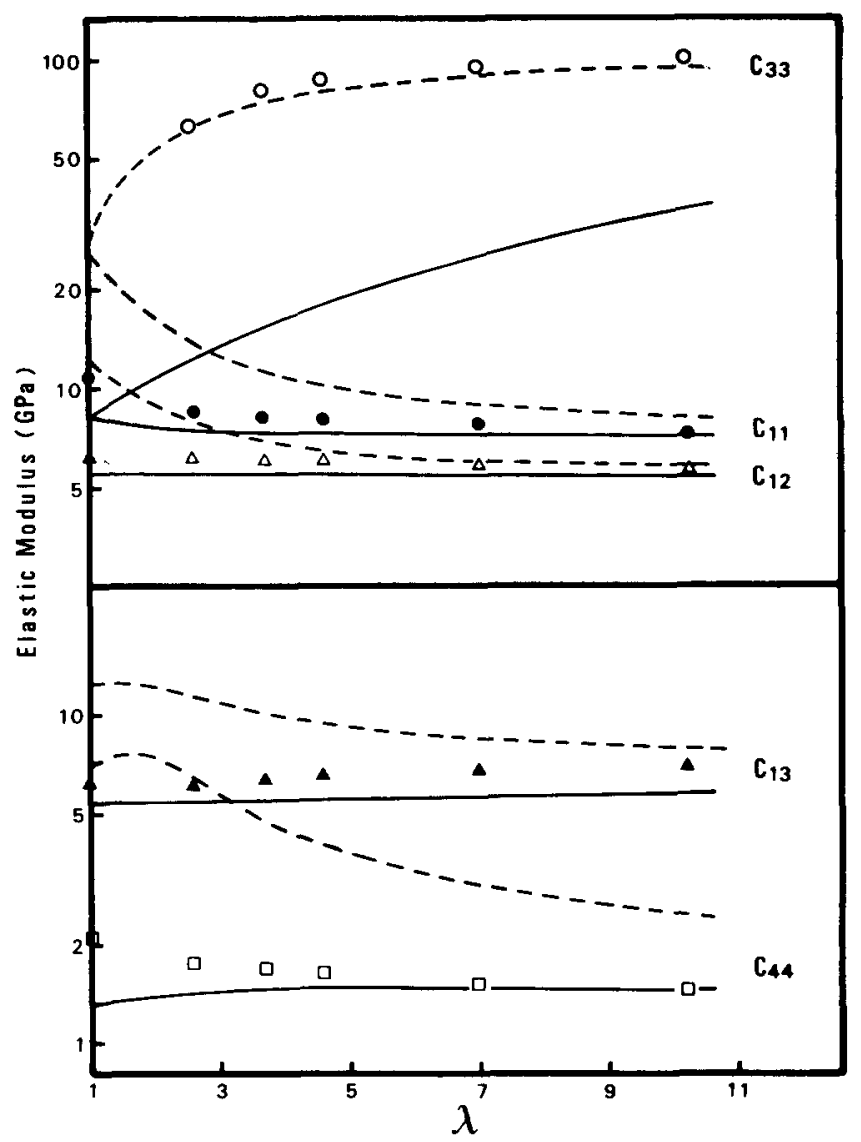

Figure 1 Draw ratio dependence of the elastic moduli of Vectra B900 at $23^{\circ} \mathrm{C}$. The Voigt (upper) and Reuss (lower) bounds calculated according to the aggregate model are shown as broken and solid lines, respectively

moduli $\left(E_{0}, E_{90}\right)$ of extruded Vectra B900 are shown as functions of draw ratio $\lambda$ in Figures 1 and 2 . The axial extensional $\left(C_{33}\right)$ and Young's $\left(E_{0}\right)$ modulus increase sharply with $\lambda$ up to about $\lambda=4$, above which they show a more gradual increase. At $\lambda=10.2, E_{0}$ has a high value $(92 \mathrm{GPa})$. In the transverse direction, the moduli $\left(C_{11}\right.$, $E_{90}$ ) exhibit a moderate drop with increasing $\lambda$. This behaviour reflects the preferential orientation of the molecular chains along the extrusion direction. Both the axial $\left(C_{44}=C_{55}\right)$ and transverse $\left(C_{66}\right)$ shear modulus decrease with increasing $\lambda$, with $C_{66}$ showing a more 


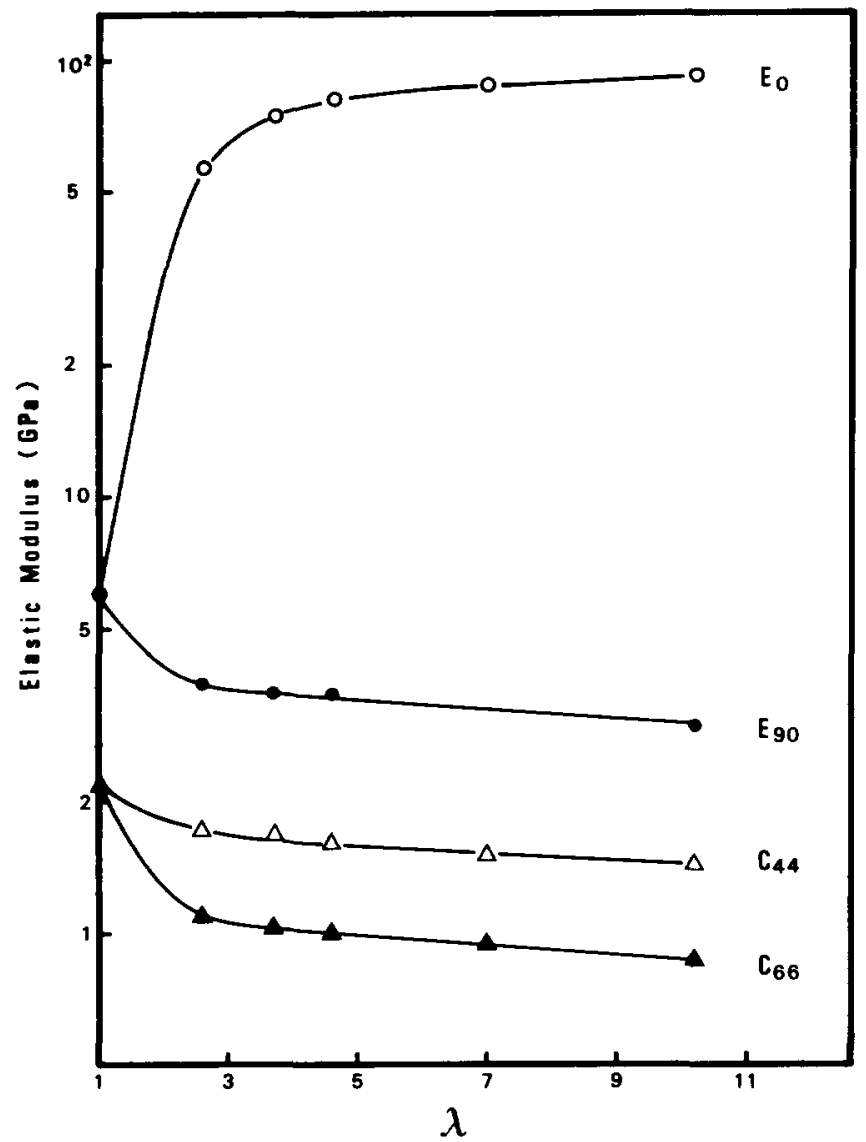

Figure 2 Draw ratio dependence of the axial $\left(E_{0}\right)$ and transverse $\left(E_{90}\right)$ Young's modulus and axial $\left(C_{44}\right)$ and transverse $\left(C_{66}\right)$ shear modulus of Vectra $\mathrm{B} 900$ at $23^{\circ} \mathrm{C}$

substantial decrease. The observed anisotropy pattern $C_{44}>C_{66}$ is also consistent with chain alignment along the extrusion direction. However, the fact that $C_{44}$ for the oriented samples is lower than the shear modulus for the isotropic material indicates that there is a shearing process occurring in the plane containing the extrusion axis. This may involve the relative sliding of the rod-like chains. The cross-plane moduli $C_{12}$ and $C_{13}$ are rather insensitive to drawing, with $C_{12}$ and $C_{13}$ showing a slight decrease and increase, respectively.

Several workers ${ }^{12-14}$ have carried out dynamic mechanical measurements $(0.3-10 \mathrm{~Hz})$ on liquid crystalline polymers with chemical composition similar to those of our samples. At $10 \mathrm{~Hz}$, the $\tan \delta$ plots show two major relaxations at $\sim 100$ and $50^{\circ} \mathrm{C}$ and a very weak relaxation around $-60^{\circ} \mathrm{C}$. The highest temperature relaxation $(\alpha$ process) is akin to a glass transition while the lower temperature $\beta$ and $\gamma$ processes are local mode processes associated with the naphthalene and phenylene moieties, respectively. Using the activation energy of the $\gamma$ relaxation obtained by Troughton et al. ${ }^{14}$ and the time-temperature equivalence, we estimate that the ultrasonic $(10 \mathrm{MHz})$ data at $23^{\circ} \mathrm{C}$ should be compared with the low frequency data below $-80^{\circ} \mathrm{C}$. We see from Table 2 that our $E_{0}$ and $C_{44}$ data at $\lambda=10.2$ agree with those of Troughton et al. on extruded HBA/HNA rods to within $10 \%$. However, the $E_{0}$ value measured by Zimmermann and Wendorff ${ }^{13}$ is much smaller, indicating perhaps a lower degree of chain orientation in their extruded film sample. We also note that their $C_{44}$ and $C_{66}$ values are even higher than the shear modulus of our unoriented sample.
It is also interesting to compare the stiffnesses of highly extruded Vectra B900 with those of high density polyethylene, which can be drawn in the solid state to give the highest axial stiffness ever achieved in flexible chain polymers. For polyethylene crystallized from the melt and then drawn 27 times, the axial stiffness $C_{33}=$ $80 \mathrm{GPa}, \sim 20 \%$ lower than that of extruded Vectra B900 $(\lambda=10.2)$. The other stiffnesses of these two polymers also have comparable values (Table 3 ). It should be noted that the highly oriented Vectra B900 sample was extruded from the melt, thus consuming a smaller amount of energy.

A comparison of the data in Table 1 and Figure 1 shows that it is easier to induce orientation in Vectra B900 than in Vectra A950. The axial stiffness $C_{33}$ for extruded Vectra $\mathrm{A} 950$ at $\lambda=3$ is $52.8 \mathrm{GPa}$ whereas Vectra $\mathrm{B} 900$ has an even higher value $(62.1 \mathrm{GPa})$ at a lower $\lambda(=2.6)$. Similar results were obtained in the earlier work of Chung ${ }^{15,16}$.

The anisotropy $C_{33} \gg C_{11}>C_{22}$ observed in the skin layer $(0.7 \mathrm{~mm}$ thick $)$ of injection-moulded Vectra A950 reveals that the molecular chains lie mostly in the plane of the bar ( $\hat{\mathbf{1}}-\hat{\mathbf{3}}$ plane) and preferentially along the injection direction ( $\hat{3}$-axis). This is consistent with the anisotropy in the thermal diffusivity observed in our previous work ${ }^{17}$ on the same sample. The high value of $C_{55}$, the modulus associated with shear deformation in the $\hat{1}-\hat{3}$ plane, and the anisotropy pattern of the shear modulus $\left(C_{55}>C_{44}>C_{66}\right)$ also reflect preferential chain orientation parallel to the $\hat{\mathbf{1}}-\hat{\mathbf{3}}$ plane. The data in Table 1 also suggest that the chain orientation in the core region is essentially random since the extensional stiffness along the thickness direction $C_{22}$ is only $20 \%$ lower than the stiffness in the $\hat{\mathbf{1}}-\hat{\mathbf{3}}$ plane.

\section{The aggregate model}

A simple theoretical framework for analysing the elastic moduli of an oriented polymer is the aggregate model $^{18}$. This model assumes that the polymer is composed of anisotropic elastic units, whose properties

Table 2 Comparison of ultrasonic modulus data (in $\mathrm{GPa}$ ) of Vectra B900 $(\lambda=10.2)$ with low frequency data of other highly oriented liquid crystalline polymers

\begin{tabular}{lllll}
\hline & $\begin{array}{l}\text { Vectra B900 } \\
23^{\circ} \mathrm{C}\end{array}$ & $\begin{array}{l}\text { HBA/HNA } \\
-80^{\circ} \mathrm{C}\end{array}$ & $\begin{array}{l}\text { HBA/HNA } \\
-80^{\circ} \mathrm{C}\end{array}$ & $\begin{array}{l}30 / 70 \\
\mathrm{D}^{a} \\
-200^{\circ} \mathrm{C}\end{array}$ \\
\hline$E_{0}$ & 92 & 104 & 102 & 60 \\
$E_{90}$ & 3.0 & & & 6.0 \\
$C_{44}$ & 1.47 & 1.6 & 1.5 & 3.4 \\
$C_{66}$ & 0.87 & & & 4.0 \\
\hline
\end{tabular}

${ }^{a}$ Polymer D contains $52 \mathrm{~mol} \%$ of HBA, $21 \mathrm{~mol} \%$ of hydroquinone, $22 \mathrm{~mol} \%$ of diphenylcarbonate and small concentrations of $4,4^{\prime}$ dihydroxydiphenyl and terephthalic acid

Table 3 Comparison of ultrasonic stiffness data (in GPa) of Vectra B900 $(\lambda=10.2)$ and high density polyethylene $(\lambda=27)$ at $23^{\circ} \mathrm{C}$

\begin{tabular}{lrrrrr}
\hline & $C_{11}$ & $C_{33}$ & $C_{13}$ & $C_{44}$ & $C_{66}$ \\
\hline $\begin{array}{l}n y y y y \\
\text { Vectra B900 }\end{array}$ & 7.26 & 100 & 7.0 & 1.47 & 0.87 \\
$\begin{array}{l}\text { High density } \\
\text { polyethylene }\end{array}$ & 6.80 & 80 & 4.7 & 1.44 & 1.48 \\
\hline
\end{tabular}

${ }^{a}$ The data for high density polyethylene are taken from reference 5 
Table 4 Calculated values (in $\mathrm{GPa}$ ) of the elastic moduli of isotropic Vectra $\mathrm{B} 900$ at $23^{\circ} \mathrm{C}$

\begin{tabular}{lcccccc}
\hline & \multicolumn{7}{c}{ Draw ratio } \\
\cline { 2 - 7 } & 1 & 2.6 & 3.7 & 4.6 & 7.0 & 10.2 \\
\hline$E$ (Reuss) & 6.02 & 4.54 & 4.39 & 4.29 & 4.00 & 3.74 \\
$E$ (Voigt) & 6.02 & 13.1 & 15.9 & 17.0 & 18.0 & 18.7 \\
$G$ (Reuss) & 2.20 & 1.63 & 1.57 & 1.53 & 1.43 & 1.33 \\
$G$ (Voigt) & 2.20 & 4.93 & 6.02 & 6.64 & 6.83 & 7.10 \\
\hline
\end{tabular}

remain constant, but are gradually aligned as the polymer is drawn or extruded. The aggregate model can be applied in two ways. First, the Reuss (uniform stress) and Voigt (uniform strain) bounds for the Young's modulus $(E)$ and shear modulus $(G)$ of the isotropic polymer can be calculated by taking the elastic constants of an oriented sample at any $\lambda$ as the intrinsic elastic constants of the microscopic unit. The comparison of the calculated values with the experimental data is shown in Table 4 . Second, the actual pattern of mechanical anisotropy can be predicted by making additional assumptions. The elastic constants of the intrinsic unit are taken to be the same as those for the most highly oriented sample $(\lambda=10.2)$. Furthermore, the development of orientation is modelled by the pseudo-affine deformation scheme, which assumes that the symmetry axes of the microscopic units rotate in the same manner as lines joining material points in the polymer. The results of these calculations are shown in Figure 1.

We see from Table 4 that both $E$ and $G$ of the isotropic sample lie within the Voigt (upper) and Reuss (lower) bounds, and are close to the Reuss bounds. Similar results were obtained for flexible chain polymers in the glassy state $^{4,6}$, indicating that the aggregate model with Reuss averaging scheme is generally applicable to polymers as long as there is very little molecular mobility in the structure.

The prediction of the development of mechanical anisotropy based on the pseudo-affine deformation scheme has a mixed success (Figure 1). While $C_{11}, C_{12}$,
$C_{13}$ and $C_{44}$ follow closely the trends predicted by the Reuss model, the increase of $C_{33}$ with $\lambda$ is much stronger than the Reuss prediction. In fact, $C_{33}$ seems to agree with the Voigt values for $\lambda>2$. This result suggests that the degree of chain alignment in the oriented sample is higher than that predicted by the pseudo-affine deformation scheme.

\section{ACKNOWLEDGEMENTS}

We are grateful to Dr T. S. Chung of Hoechst-Celanese Co. for supplying the Vectra A950 samples, and to $\mathrm{Mr}$ Bruce Bassett for preparing extruded Vectra B900 samples.

\section{REFERENCES}

1 Ciferri, A. and Ward, I. M. (Eds) 'Ultrahigh Modulus Polymers', Applied Science, London, 1979

2 Ward, I. M. (Ed.) 'Developments in Oriented Polymers1', Applied Science, London, 1982

3 Zachariades, A. E. and Porter, R. S. (Eds) 'High Modulus Polymers', Dekker, New York, 1987

4 Leung, W. P., Chen, F. C., Choy, C. L., Richardson, A. and Ward, I. M. Polymer 1984, 25, 447

5 Choy, C. L. and Leung, W. P. J. Polym. Sci, Polym. Phys. Edn. $1985,23,1759$

6 Leung, W. P., Chan, C. C., Chen, F. C. and Choy, C. L. Polymer $1980,21,1159$

7 Choy, C. L., Leung, W. P. and Huang, C. W. Polym. Eng. Sci. $1983,23,910$

8 Leung, W. P., Choy, C. L., Nakagawa, K. and Konaka, T. J. Polym. Sci., Polym. Phys. Edn. 1987, 25, 2059

9 Leung, W. P. and Choy, C. L. J. Polym. Sci., Polym. Phys. Edn. $1983,21,725$

10 Crevecoeur, G. and Geoeninckx, G. Polym. Eng. Sci. 1990, 30, 532

11 Bassett, B. R. and Yee, A. F. Polym. Comp. 1990, 11, 10

12 Blundell, D. J. and Buckingham, K. A. Polymer 1985, 26, 1623

13 Zimmermann, H. J. and Wendorff, J. H. J. Mater. Sci. 1988, 23, 2310

14 Troughton, M. J., Davies, G. R. and Ward, I. M. Polymer 1989, 30, 58

15 Chung, T. S. J. Polym. Sci., Polym. Lett. Edn. 1986, 24, 299

16 Chung, T. S. J. Polym. Sci., Polym. Phys. Edn. 1988, 26, 1549

17 Choy, C. L., Leung, W. P. and Kwok, K. W. Polym. Commun. 1991, 32, 285

18 Ward, I. M. Proc. Phys. Soc. 1962, 80, 1176 